\title{
LOS TÉRMINOS DE INTERCAMBIO EN COLOMBIA: TEORÍA Y EVIDENCIA
}

\author{
EDGARDO DE LA CRUZ DEL VILLAR \\ Profesor \\ Facultad de Ciencias Económicas \\ Universidad de Cartugena
}

\section{LA TEORIA}

T a evidencia acumulada en los años recientes ha venido a corroborar la hipótesis seminal de Prebisch- Singer, según la cual, los precios reales de las materias primas y alimentos tienden a deteriorarse en el largo plazo (Spraos 1983, Sapsford 1985, Sarkak 1986, Difilippo 1987, Scandizzo y Diskosaws 1987 y Grilli y Yang $1988^{1}$

Este fenómeno, se argumenta, dificulta las posibilidades de desarrollo de la periferia. Se dice, existe, una estrecha relación entre el ciclo económico y los movimientos en los términos de intercambio.

Un aumento en los términos de intercambio incrementa el ahorro privado e influye a su vez en la cuenta corriente de la Balanza de Pagos ${ }^{2}$

De igual manera una disminución en los términos de intercambio, produciría el efecto contrario.

${ }^{1}$ OCAMPO José Antonio. Los Términos de Intercambio y las Relaciones Centro Periferia. El Desarrollo desde dentro. El Trimestre Económico F.C.E. abril de 1.995.

${ }^{2}$ GAVIRIA Alejandro. El ahorro privado y los términos de intercambio. El caso Colombiano. Revista Ensayos sobre Política Económica. Banco de la República. Junio de 1993. 
Los términos de Intercambio (T.D.I), afectan también los ingresos fiscales, la inversión, la tasa de cambio, el consumo y condiciona la política económica para contrarrestar sus efectos. En Colombia los términos de intercambio (T.D.I) han estado intimamente asociados a los precios internacionales del café, debido a su alta participación en los ingresos externos del país. En ocasiones han compensado los efectos desfavorables de los Shock Externos ${ }^{3}$

Así mismo, Colombia y América Latina, en su conjunto, durante más de medio siglo, han orientado la definición de su estrategia de desarrollo hacia la sustitución de importaciones de carácter manufacturero, como una respuesta, inicialmente expontánea y luego deliberada, al deterioro radical de los precios internacionales de los productos primarios, entre otras causas. $^{4}$

3 CARDENAS Mauricio. Ciclos Económicos y Bonanzas Exportadoras. Revista Ensayos sobre Política Económica. Junio 1992. Robert STEINER. Ajustando el desequilibrio en la cuenta, nueva evidencia para Colombia. Revista Ensayos sobre Política Económica.

4 LYNN GROUND RICHARD. La Génesis de la Sustitución de Importaciones en América Latina. Revista CEPAL $\mathrm{N}^{\circ}$ 36.Dic. 1988.
Sin embargo, recientemente, algunas teorías sobre los T.D.I, han dado más importancia a las características de los países, antes que a los bienes que producen, como causa del deterioro de los T.D.I. Así se sostiene, que las manufacturas de países de menor desarrollo, también están sometidas al deterioro de los precios en la relación de intercambio, aunque con una menor intensidad que en los productos agrícolas ${ }^{5}$ Sería importante, examinar la evolución histórica de los precios de los productos manufacturados objetos de exportación a fin de confirmar esta formulación teórica para Colombia.

No obstante la numerosa bibliografia sobre la caída secular de los TDI en el largo plazo, recientemente, dos funcionarios del Banco Mundial y el Banco Interamericano de desarrollo, utilizando series de TDI de la CEPAL (1928-93) y utilizando técnicas econométricas no paramétricas, diferentes a las usualmente utilizadas, encontraron que los datos y conclusiones a las que llegaron no confirman la evidencia empírica de la hipótesis Prebisch-Singer, en el sentido de una caída en los precios relativos de

${ }^{5}$ OCAMPO José.Op Cit. 
los productos primarios con respecto a los de manufacturas ${ }^{6}$.

También refutan la idea generalizada de que la mayoría de los países de la periferia enfrentan T.D.I impredecibles. Sostienen, además, que una de las limitaciones de los estudios que confirman la hipótesis Prebisch-Singer, obedece al uso de metodologías econométricas que no permiten una adecuada medición de la tendencia de largo plazo y de la persistencia de los choques, debido a que se le da demasiada importancia a características dinámicas de corto plazo $^{7}$

Durante largos períodos los índices de la relación de precios de intercambio de Colombia se podrán interpretar como una relación entre los precios de las exportaciones de productos primarios y los precios de las importaciones de manufacturas. Los cambios, sin embargo, de composición que han ido ocurriendo en el comercio exterior colombiano, deben reflejarse en cambios en los índices T.D.I como consecuencia de la influencia que ejercen los nuevos productos que integran dicho comercio,

${ }^{6}$ LEON Javier y SOTO Raimundo. Términos de Intercambio en América Latina. El Trimestre Económico. AbrilJunio 1995.

${ }^{7}$ LEON y SOTO, Op Cit particularmente para el caso colombiano, la influencia que ejercen los productos mineros y las manufacturas. En consecuencia, en épocas recientes debería presentarse una mejoría en los T.D.I para Colombia.

\section{LA EVIDENCIA}

a) Sustitución de Importaciones. La crisis mundial llegó a Colombia, en medio de una bonanza externa sin precedentes. Todos los signos fueron excepcionalmente positivos hasta 1928: las exportaciones de café y de petróleo estaban en plena expansión, la relación de precios de intercambio era muy favorable, especialmente para el café, el país estaba recibiendo del exterior capitales en forma masiva y las importaciones reales se encontraban en su punto mas alto ${ }^{8}$ "

Este pasaje refleja la situación que vivía el país en el segundo quinquenio de los años veinte. Era una época de aumento considerable de inversiones, de rápida acumulación de capitales, con una balanza comercial casi siempre favorable durante toda la década,

8 OCAMPO Antonio, José, MONTENEGRO Santiago. "La Crisis Mundial de los años treinta en Colombia" Crisis Mundial, Protección e Industrialización CEREC FESCOL 1984. 
Esto último, parecer ser, ocurrió en Colombia, entre 1990-1996. La gráfica siguiente sobre indice de términos de intercambio, muestra que los términos de intercambio sufrieron una declinación entre 1990-1994 con una leve recuperación en el segundo semestre de este último año para luego declinar nuevamente hasta 1996.

\section{Índice de la Relación de Términos de Intercambio ${ }^{14}$}

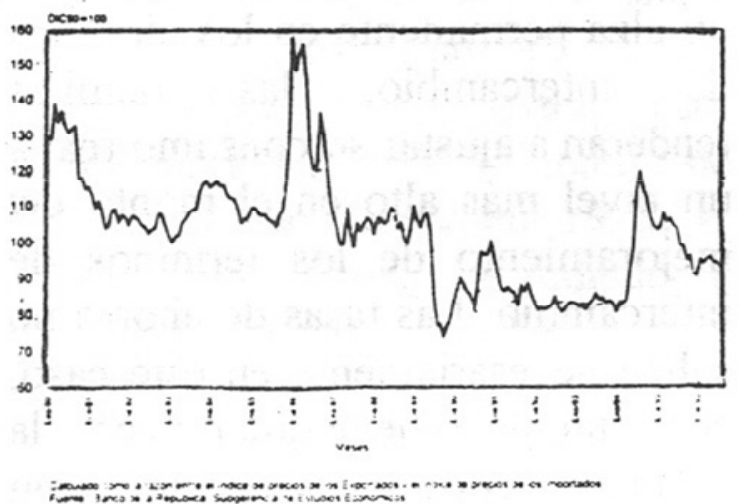

Como se observa en la gráfica mencionada, la tendencia de los T.D.I en los años ochenta tenía un nivel más alto que en los noventa, estaba encima del índice de 100 para pasar a un nivel menor, ligeramente superior a 80 , perdiendo aproximadamente 20

14 Gráfico presentado por Alberto Carrasquilla Barrera en Seminario de Verano sobre "Tópicos de Teoría y Política Macroeconómica colombiana. Universidad Jorge Tadeo Lozano del Caribe. Cartagena julio 1997. puntos entre una década y otra (80'y $\left.90^{\prime}\right)$.

Este deterioro de los T.D.I en los noventa, podría estar asociado con la caída del ahorro privado desde 1990 hasta 1994, para mejorar ligeramente en 1995 y 1996, lo cual, parecería estar relacionado con la mejoría de los T.D.I entre mediados de 1994 y 1996. (compare gráfica de T.D.I con la gráfica del ahorro).

Colombia Ahorro Privado ${ }^{15}$

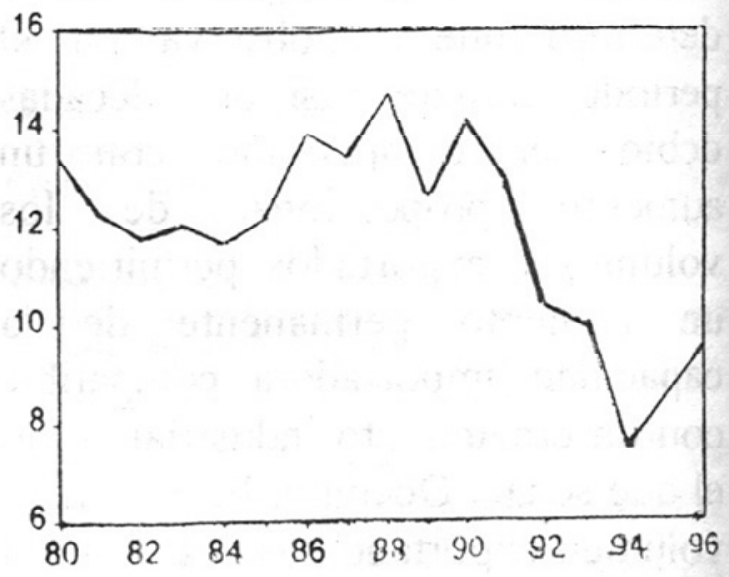

Causa la impresión que el resultado macroeconómico está confirmando la teoría antes expuesta. En efecto las tasas de ahorro fueron bajas entre 1980-1990. El promedio marcó el $13 \%$. En los noventa se situaron a niveles por debajo del $10 \%$, como se observa en la gráfica,

15 Gráfico presentado por Alberto Carrasquilla Barrera en Seminario de Verano sobre "Tópicos de Teoría y Política Macroeconómica colombiana. Universidad Jorge Tadeo Lozano del Caribe. Cartagena julio 1997. 
con acumulación de reservas internacionales, de grandes volúmenes de importación de bienes de capital y de aparición de cientos de empresas de gran tamaño.

Según Ocampo y Montenegro, desde 1922 hasta 1928, la relación de precios de intercambio (T.D.I) de Colombia fue creciente y favorable y dependió de los buenos precios de solo dos productos de exportación, café y petroleo ${ }^{9}$. A partir de 1928 comenzó a declinar hasta los años cuarenta, luego mejoró ligeramente para caer nuevamente en 1945. El índice de T.D.I pasó de $108 \%$ en 1928 , el más alto del período estudiado, a $60 \%$ en 1940 , perdiendo 48 puntos $^{10}$

Como lo demuestran varios autores colombianos e instituciones extranjeras, Ocampo, Rodriguez, Cepal, entre otros, coinciden en afirmar que la industria colombiana creció entre 1931-39 a niveles nunca vistos, $12.4 \%$ (Ocampo Montenegro), en el mismo período en que los T.D.I de Colombia caían dramáticamente.

9 La composición de las Exportaciones entre $1925-29$ era de $85.1 \%$ (Café y Petróleo) y en 1950-53 había aumentado a 94.7\% (Café y Petróleo).

${ }^{10}$ OCAMPO José Antonio, Op Cit.
Este deterioro de los T.D.I y el proceso de surgimiento y consolidación de la industria colombiana, también estaría comprometido con una estrategia de sustitución de importaciones, como se observa en el siguiente cuadro:

\section{Composición de las Importaciones Colombianas 1925- $1953^{11}$ $\%$ Periodos}

\begin{tabular}{|c|c|c|c|c|}
\hline & $1925-29$ & $1930-38$ & $1939-45$ & $1945-53$ \\
\hline Total & 100 & 100 & 100 & 100 \\
\hline Bienes de consum o & 43.5 & 45.4 & 30.1 & 22.5 \\
\hline $\begin{array}{c}\text { Maquinaria y } \\
\text { Equipo Productivo }\end{array}$ & 32.2 & 21.4 & 21.6 & 36.6 \\
\hline $\begin{array}{c}\text { Materias Prim as y } \\
\text { Productos } \\
\text { Intermedios }\end{array}$ & 24.3 & 33.2 & 48.3 & 49.9 \\
\hline
\end{tabular}

$\mathrm{Si}$ la tesis de Lynn Ground ${ }^{12}$ es cierta, el fuerte deterioro de los T.D.I, que se observó en el país en el período señalado, obligó a desarrollar mecanismos para defenderse de esta situación adversa y adoptar una política de sustitución de importaciones.

En efecto, entre 1925-20 la importación de bienes de consumo era de $43.5 \%$ la de maquinaria y equipo: $32.2 \%$ y la de materias

11 LLERA R. Carlos. Comercio Internacional. Edit. Bedout 1965.

${ }^{12}$ LYNN Richard Ground. OP. Cit 
primas: $24.3 \%$; transcurridos veinticinco años, en el período 1945-53 la estructura de las importaciones se había modificado así: bienes de consumo $22.5 \%$ maquinaria y equipo: $36.6 \%$ y $40.9 \%$. Esta variación radical de la estructura de las importaciones estaría revelando un proceso de sustitución de bienes de consumo por parte de la industria que se instaló y desarrolló desde 1925 hasta 1950, lo cual parecería confirmar las tesis de Lynn Ground que a su vez proviene de SingerPrebisch. Sin embargo, el fuerte deterioro que se observa en el período dùrante casi dos décadas debió ser compensado con un aumento proporcional de los volúmenes exportados permitiendo un aumento permanente de la capacidad importadora compatible con un crecimiento industrial como el que señala Ocampo. En efecto, el volumen exportado pasó de $373 \mathrm{mil}$ ton, en 1925 , a 4.5 millones de tons en 1950, es decir, aumentó casi 12 veces compensando con creces la caída en el índice de T.D.I.

\section{b) La Cuenta Corriente, el} Ahorro y la Inversión Privada.

La otra evidencia, que parecería estar asociada con la caída de los T.D.I, es mas reciente, y está relacionada con el ahorro privado, la cuenta corriente de la balanza de pagos y la inversión, en lo que va transcurrido de los años noventa en Colombia.

La teoría sobre los T.D.I, sostiene que "un alza transitoria en los términos de intercambio implica también un incremento transitorio del ingreso. En consecuencia, el ahorro agregado en el país tenderá a subir debido a la estabilidad del consumo. La cuenta corriente tenderá a moverse hacia un superávit. Sin embargo, después de un alza permanente en los términos de intercambio, las familias tenderán a ajustar su consumo real a un nivel más alto en el monto del mejoramiento de los términos de intercambio. Las tasas de ahorro no suben necesariamente en este caso, como tampoco tiene que moverse la cuenta corriente hacia un superávit. ${ }^{13}$

En igual forma, un deterioro en los términos de intercambio (T.D.I) debe conducir a una reducción transitoria del ingreso, el ahorro caerá y la cuenta corriente reflejará un déficit. Además, como el consumo es función del ingreso permanente, una caída súbita y transitoria en el ingreso corriente, origina endeudamiento para "suavizar" el patrón de consumo.

${ }^{13}$ SACHS Larrain. Macroeconomía en la Economía Global. Prentice Hall. Hispanoamericana S.A. 1994. 
sobre el ahorro como consecuencia de la declinación de aproximadamente 20 puntos en los indices de T.D.I.

Caso contrario ocurrió con la bonanza cafetera del segundo quinquenio de los setenta (75-79) en donde el ahorro interno subió como proporción del P.I.B y la cuenta corriente mejoró ostensiblemente

La evidencia empírica para la cuenta corriente de la Balanza de Pagos entre 1990-1996, como se observa en la gráfica siguiente, pareceria estar confirmando también la teoría. En efecto, la gráfica muestra, que después de un período de precios altos del café (1975-1979), donde éstos alcanzaron los mayores niveles de toda la historia cafetera del país (el nivel más alto alcanzó casi US $\$ 3.00$ la libra), se inicia un descenso de los precios cafeteros y una caída dramática de los términos de intercambio $\mathrm{y}$, paralelamente, la cuenta corriente de la balanza de pagos muestra también un déficit de una proporción similar a la caída de los T.D.I ${ }^{16}$

${ }^{16}$ Sin embargo para otros autores, el fuerte déficit de la cuenta corriente de la Balanza de Pagos podrá estar asociada a la política de liberación de importaciones que se ensayó en ese período para disminuir el crecimiento considerable de las Reservas internacionales y contrarrestar la presión
Nuevamente la recuperación de la cuenta corriente desde 1985, parece estar relacionada con un mejoramiento de los T.D.I como consecuencia de los mejores precios del café en esos mismos años. Finalmente, nuevamente la caída de los T.D.I al iniciarse los noventa se refleja en la caída en picada de la cuenta corriente de la balanza de pagos por último, iniciar una muy sutil tendencia al ascenso.

\section{Cuenta Corriente de la Balanza de Pagos ${ }^{17}$}

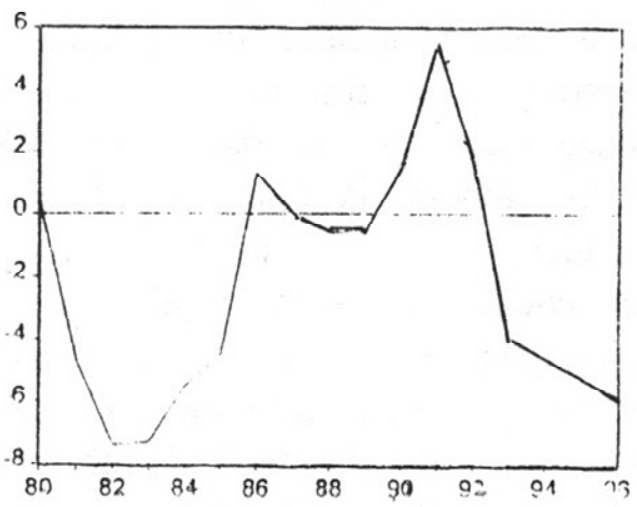

Como el ahorro privado cayó, la inversión sólo pudo financiarse a través del ahorro externo (ver cuadro siguiente). En efecto, la deuda externa privada pasó de 2.7 millones de dólares en 1992 a 13 millones de dólares en 1996.

hacia la revaluación de la moneda (Luis Jorge Garay)

17 Gráfico presentado por Alberto Carrasquilla Barrera en Seminario de Verano sobre "Tópicos de Teoría y Política Macroeconómica colombiana. Universidad Jorge Tadeo Lozano del Caribe. Cartagena julio 1997 


\section{Inversión}

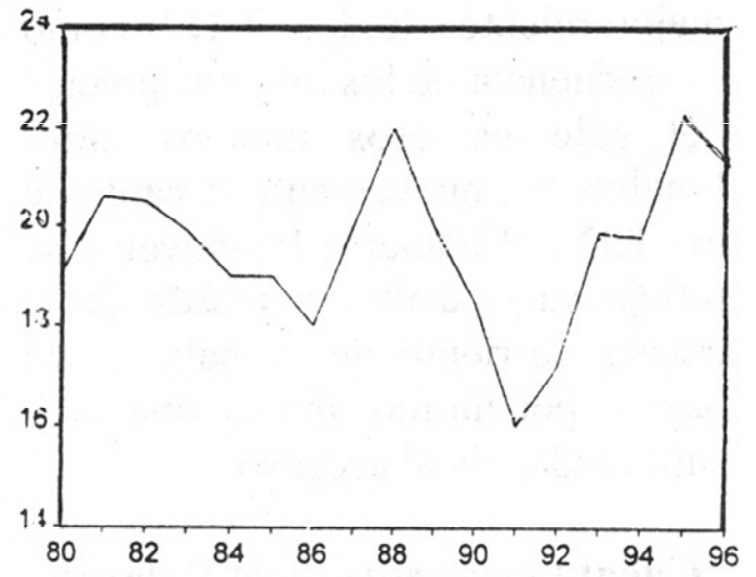

La inversión, a pesar que el ahorro privado interno cayó estrepitosamente, se mantuvo en los niveles tendenciales de las últimas décadas: $19-20 \%$. Incluso, estuvo por encima de este nivel 2 ó 3 puntos en 1995 - 1996. La teoría supone que la inversión debió haber caído si el régimen cambiario hubiese continuado con rígidos controles a la entrada de capitales y en este caso se habría confirmado la teoría.

De acuerdo con lo anterior la evidencia de los años 1925-1949 en relación con la influencia de los T.D.I en la estrategia de sustitución de importaciones y la mas reciente de los años 1990 - 1996 con respecto a el ahorro privado a la inversión privada y a la cuenta corriente de la balanza de pagos, parecería estar confirmando las dos versiones de la teoría sobre los T.D:I.

\section{BIBLIOGRAFIA}

OCAMPO José Antono, MONTENEGRO Santiago. "La Crisis Mundial de los años treinta en Colombia", Crisis Mundial, protección e Industrialización. CEREC FESCAL 1984.

GAVIRIA Alejandro. El Ahorro Privado y los Términos de Intercambio. El caso Colombiano. Revista Ensayos sobre Politica Económica .Banco de la República. Junio de 1993.

LLERAS R. Carlos Comercio Internacional. Edit Bedout 1965.

SACHS -LARRAIN. Macroeconomía en la Economía Global. Prentice Hall. 1994.

LYNN GROUND Richar. La Génesis de la sustitución de importaciones en América Latina. Revista CEPAL $N^{\circ}$ 36. Diciembre 1988.

LEON Javier, SOTO Raimundo. Términos de Intercambio en América Latina. El trimestre Económico. Abril, Junio de 1995. 